\title{
Robust Control of a Two-Mass-Spring System subject to its Input Constraints
}

\author{
Wolfgang Reinelt \\ Department of Electrical Engineering \\ Linköping University, S-581 83 Linköping, Sweden \\ WWW: http://Www. control.isy.liu.se/ ${ }^{\sim}$ wolle/ \\ Email: wolle@isy.liu.se
}

August 1999, updated February 2000

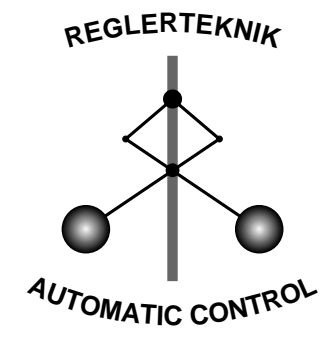

LINKÖPING

Report no.: LiTH-ISY-R-2201

Accepted for presentation at American Control Conference, Chicago, USA.

28-30 June 2000. Session Code: TA13-1

Technical reports from the Automatic Control group in Linköping are available by anonymous ftp at the address ftp.control.isy.liu.se. This report is contained in the portable document format file 2201.pdf. 


\title{
Robust Control of a Two-Mass-Spring System subject to its Input Constraints
}

\author{
Wolfgang Reinelt \\ Division of Automatic Control, Dept of Electrical Engineering, \\ Linköping University, 58183 Linköping, Sweden, \\ email: wolle@isy.liu.se, http://www.control.isy.liu.se/ wolle/
}

\begin{abstract}
A robust controller is designed for a system consisting of two carts coupled by a spring. Special attention is given to the input saturations. This problem was posed within a benchmark collection for robust control techniques and treated for example at the 1992 ACC. $\mu$ analysis and simulation studies illustrate our design.
\end{abstract}

Keywords: Robust Control, $\mathcal{H}_{\infty}$ Loop Shaping, Hard Constraints, Saturations, Two-Mass-Spring System.

\section{Introduction and Motivation}

Most practical control problems are dominated by hard bounds. Valves can only be operated between fully open and fully closed, pumps and compressors have a finite throughput capacity and tanks can only hold a certain volume. These input- or actuator-bounds convert the linear model into a nonlinear one. Exceeding these prescribed bounds causes unexpected behavior of the system - large overshoots, low performance or (in the worst case) instability. Solving control problems subject to hard bounds, we need to restrict the amplitudes of the external signals (i.e. the reference signals) as well. In this approach, we regard reference signals, bounded in amplitude and speed (i.e. the first derivative has to be bounded in its amplitude). This appears in many systems, for example in a tank, not only the liquid-level is bounded (by the tanks height), additionally the liquid cannot change its level arbitrarily fast.

Design of controllers for systems with hard constraints is a quite vivid area of research, see for example the recent textbook [10] or the overview paper [1] and the references therein. A rather general and unified description of Anti Windup schemes is given in [5].

Furthermore, process models are always inaccurate. Even when having an extremely detailed model, the real plant may contain unknown or slowly changing physical parameters. Thus, the controller has to manage the difference between the model (used for design) and the real plant, to which the controller is applied in practice. Bridging the gap between model and real plant is the field of robust controller design.

Quite a huge amount of literature is available on robust control. We therefore only refer to the survey paper [3] and standard textbooks on $\mathcal{H}_{\infty}$ control techniques $[4,6,13]$ which we will be using in this work.

In order to study different methods for designing robust controllers, a benchmark collection has been set up [12]. The task is to control a SISO system, consisting of two masses coupled by a spring, under certain circumstances. One of these problems reflects the practical situation of having a bounded control signal. This problem has also been studies for example in $[2,11]$ using K-D iteration and $\nu$-gap techniques respectively. We apply the $\mathcal{H}_{\infty}$ Loop Shaping Design Procedure (LSDP) to this problem, indeed an extension of this procedure in order to meet the prescribed hard bound for the control signal. We review this procedure in sec. 2. One main point is the systematic adjustment of the design weights during the loop shaping procedure in the case that the bounds are not met. We derive an explicit relation between maximum control variable and the singular values of the corresponding transfer function, visible during the loop shaping procedure. This design philosophy has already been outlined in [8]. One theoretical contribution of this work is the extension of the result on weight adaption to arbitrary systems (the restriction to strictly proper systems, as made in [8], is no longer necessary). This is discussed in sec. 3. 


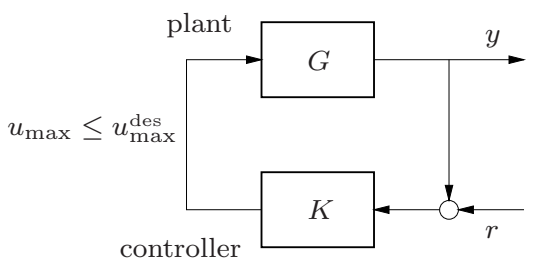

Figure 1: Control loop with constraint control variable $u$.

The two mass spring system serves as an example for this design procedure. The problem itself is described in sec. 4 . We suggest two designs in sec. 5 and study them using $\mu$ analysis. Simulation studies in sec. 6 illustrate these designs. We summarize the work in sec. 7, where future research is indicated as well.

\section{$2 \quad \mathcal{H}_{\infty}$ Loop Shaping for Systems with Constraints}

We study control systems with reference signals, bounded in amplitude and speed. Aim of the controller design is to handle hard bounds of the control signal. The signals are depicted in the standard control loop in fig. 1. We start with the following definitions, given in [7]. An extension to the multivariable case is possible [8], but not needed to solve the benchmark example.

2.1 Definition (Admissible Reference Signal) Let $R, \dot{R}>0$. Then a reference signal $r$ is called $(R, \dot{R})$ admissible, when $|r(t)| \leq R$ and $|\dot{r}(t)| \leq \dot{R}$ hold for all $t>0$. The set of all $(R, \dot{R})$-admissible reference signals is denoted by $\mathcal{A}(R, \dot{R})$.

2.2 Definition (Maximum Control Signal) Given the internally stable standard control loop as in fig. 1. Then the Maximum Control Signal is defined by

$$
u_{\max }:=\sup \left\{\|u\|_{\infty} ; \forall r \in \mathcal{A}(R, \dot{R})\right\}
$$

The definition of the admissible reference signal is quite straightforward from the motivation. We state the following result given by Reichel [7]:

2.3 Theorem and Algorithm (Calculation of the Maximum Control Signal) Given a linear and time invariant multivariable control system with reference signal $r(t)$ and control signal $u(t)$. Let the reference signal be $(R, \dot{R})$-admissible. Then:

(a) There exists an algorithm that determines the maximum control signal $u_{\max }$ according to Definition 2.2 of this system for all admissible reference signals.

(b) An $(R, \dot{R})$-admissible input exists, so that $u_{\max }$ is achieved.

The algorithm outlined in the original work [7] constructs a worst case input $r$. An alternative numerical solution of the problem, based on nonlinear optimization, has been shown in [9]. Independent of the numerical solution, Theorem 2.3 can be used to determine the maximum control signal $u_{\max }$, when the reference signal $r(t)$ is $(R, \dot{R})$-admissible.

The result presented above enables us to calculate the maximum control signal of a control system, when the external signal, i.e. the reference signal fulfills these constraints. We will exploit this to extend the $\mathcal{H}_{\infty}$ Loop Shaping Design Procedure by McFarlane \& Glover [6] to the case of constraint control signals:

Given plant $G$, restrictions $R, \dot{R}>0$ for the reference signal and a desired maximum control signal $u_{\max }^{\text {des }} \succ 0$ we will perform the Loop Shaping Design Procedure (LSDP) in the following way:

1. Choose a performance factor $f$ and weight $W$ to shape the plant.

2. Controller-design for the shaped plant and calculation of the stability margin $\epsilon$.

3. Calculation of the final controller (including the weight).

4. Decide whether the design-objectives are fulfilled or not: 
- Is the stability margin $\epsilon$ large enough?

- Are the performance-objectives fulfilled?

- Does $u_{\max } \leq u_{\max }^{\text {des }}$ hold?

If not, choose other weight $W$ (and/or another performance factor $f$ ) and go back to the first step.

Theorem 2.3 enables us to determine the maximum control signal $u_{\max }$ within the control loop, which allows the check maximum control signal in step 4. This additional check is the only difference to the classical LSDP. We refer to this procedure as Extended Loop Shaping Design Procedure (ELSDP).

\section{Adjustment of the Design Weights}

We discuss a question neglected in the last section: after a first choice of weights, our design objectives are usually not fulfilled - the weights have to be adjusted. In the case of a too small stability margin $\epsilon$ the strategy is clear from the classical LSDP: because there is no explicit relation known between achieved stability margin and weights, we have to examine the singular values of the shaped plant and the achieved open loop. In the frequency range with a significant difference, our weights are incompatible with the plant and have to be adjusted.In the following we discuss the remaining open question: Is there a strategy for correct and systematic adjustment of the weights, when the control signal is still too large after a loop shaping step?

Within loop shaping, we work on the singular values of different interesting transfer functions. Thus we are searching for a relation between the singular values of the transfer function and the maximum control signal $u_{\max }$. The relation between reference signal and control signal is given by $u(s)=H(s) \cdot r(s)=$ $K(s)(1-G(s) K(s))^{-1} \cdot r(s)$ and $u(t)=h(t) \star r(t)$ respectively, where " $\star$ " denotes convolution and a state space representation is given by $H=(A, B, C, D)$. In [8], we showed the following useful relation for the case of a stable and strictly proper transfer function $H:\|u\|_{\infty} \leq 2 n \cdot\|H\|_{\infty} \cdot\|r\|_{\infty}$, where $n$ denotes the McMillan degree of $H$. We extend this to the case of proper transfer functions:

3.1 Theorem In the case of a stable and proper transfer function $H$ with input $r$ and output $u$,

$$
\|u\|_{\infty} \leq\left(2\|H\|_{\infty}+3 d\right) \cdot n \cdot\|r\|_{\infty}
$$

holds with $D=\left[d_{1}, \ldots, d_{n}\right]^{T}$ and $d:=\max _{i}\left|d_{i}\right|$.

Proof. Let $\tilde{H}:=H-D=(A, B, C, 0)$. Then $h(t)=\tilde{h}(t)+D \delta(t), \delta(t)$ the $\delta$-impulse. Then $\|h\|_{1}=$ $\sum_{i=1}^{n} \int_{0}^{\infty}\left|h_{i}(t)\right| d t=\sum_{i=1}^{n} \int_{0}^{\infty}\left|h_{i}(t)-d_{i} \delta(t)+d_{i} \delta(t)\right| d t \leq \sum_{i=1}^{n}\left(\int_{0}^{\infty}\left|h_{i}(t)-d_{i} \delta(t)\right| d t+\int_{0}^{\infty}\left|d_{i} \delta(t)\right| d t\right) \leq$ $\sum_{i=1}^{n} \int_{0}^{\infty}|\tilde{h}(t)| d t+n \max _{i}\left|d_{i}\right|=\|\tilde{h}(t)\|_{1}+n d$ and therefore $\|u\|_{\infty} \leq\|h\|_{1}\|r\|_{\infty} \leq\left[\|\tilde{h}\|_{1}+n d\right]\|r\|_{\infty} \leq$ $\left[2 n\|\tilde{H}\|_{\infty}+n d\right]\|r\|_{\infty}=\left[2 n\|H-D\|_{\infty}+n d\right]\|r\|_{\infty} \leq 2 n\|H\|_{\infty}\|r\|_{\infty}+3 n d\|r\|_{\infty}$.

We now turn back to our final aim: the relation between the singular values of $H$ and $\|u\|_{\infty}$. As the control loop is internally stable, the transfer function $H$ is stable. Following equation (2), we see that decreasing the $\infty$-norm of $H(s)$ decreases an upper bound for the maximum control signal.

Suppose, the maximum control signal is too high after a loop shaping step. We then have to decrease the maximum singular value of $H$ in the frequency range where the $\infty$-norm appears. In the case of a too low maximum control signal, we have to increase the maximum singular value in that frequency range. We point out, that this affects only an upper bound for the maximum control signal. In general, there is much space between the both sides of eqn.(2). We only use it as a guideline for the adjustment of the weights in the "correct direction" and in the correct frequency range. The practical value of this guideline is shown in the example. However, the initial choice of the weights should attack the general shape of the open loop and is discussed in context with original Loop Shaping Design Procedure by McFarlane \& Glover [6] and textbooks on robust control $[4,13]$. 


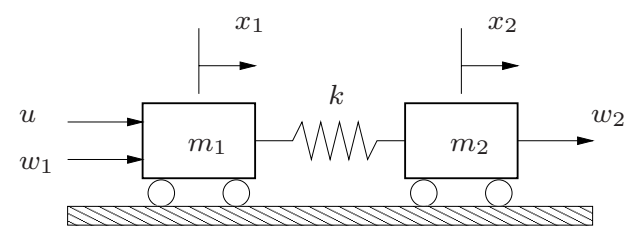

Figure 2: Two mass spring system with uncertain parameters.

\section{Benchmark Problem}

We consider the two mass spring system shown in figure 2. It is assumed that for the nominal system $m_{1}=m_{2}=1$ and $k=1$ hold. The control force $u$ acts on body 1 , while the position of body 2 is measured. The system can be represented in the state-space form:

$$
\begin{aligned}
{\left[\begin{array}{l}
\dot{x}_{1} \\
\dot{x}_{2} \\
\dot{x}_{3} \\
\dot{x}_{4}
\end{array}\right]=} & {\left[\begin{array}{cccc}
0 & 0 & 1 & 0 \\
0 & 0 & 0 & 1 \\
-k / m_{1} & k / m_{1} & 0 & 0 \\
k / m_{2} & -k / m_{2} & 0 & 0
\end{array}\right]\left[\begin{array}{l}
x_{1} \\
x_{2} \\
x_{3} \\
x_{4}
\end{array}\right] } \\
& +\left[\begin{array}{c}
0 \\
0 \\
1 / m_{1} \\
0
\end{array}\right]\left(u+w_{1}\right)+\left[\begin{array}{c}
0 \\
0 \\
0 \\
1 / m_{2}
\end{array}\right] w_{2} \\
y= & x_{2}+v \\
z= & x_{2} \quad \text { (controlled output) }
\end{aligned}
$$

or as transfer-function:

$$
G(s)=\frac{k}{m_{1} m_{2} s^{4}+k\left(m_{1}+m_{2}\right) s^{2}}=\frac{z(s)}{u(s)}
$$

The aim of the benchmark collection, is to design a linear feedback-controller with constant gain. We focus on the following task:

Benchmark Problem \#4 [12]. Unit step output command tracking for the controlled output $z(t)$ with the following properties:

1. control input is limited to $|u| \leq 1$

2. performance: settling time and overshoot are both to be minimized

3. robustness: performance and stability with respect to all uncertain parameters are both to be maximized

4. consider conflicts between the last two properties

This benchmark problem is a robust control problem with real uncertainties. Speaking in terms of $\mathcal{H}_{\infty^{-}}$ control theory, it is a problem with structured uncertainties. We want to examine our controller designs, done in the next section, for robust stability. I.e. for which variations of the three uncertain parameters remains the closed system stable? An appropriate tool for analyzing this is the $\mu$-test, see [13]. Therefore, we formulate this problem in terms of $\mu$-analysis.

Fig. 3 shows the plant with respect to its uncertain parameters $k, m_{1}, m_{2}$. To carry out the $\mu$-analysis, the constant gains $k, \frac{1}{m_{1}}, \frac{1}{m_{2}}$ are replaced by the blocks shown in figure 4, using the following Lower Linear Fractional Transformations (LLFTs) for the perturbed parameters:

$$
\begin{aligned}
\frac{1}{m_{i, \text { pert }}} & =\mathcal{F}_{L}\left(\frac{1}{m_{i, \text { nominal }}}\left[\begin{array}{cc}
1 & -\gamma_{i} \\
1 & -\gamma_{i}
\end{array}\right], \delta_{i}\right) \\
& =: \quad \mathcal{F}_{L}\left(\mathcal{M}_{i}, \delta_{i}\right)=\frac{1}{m_{i, \text { nominal }}+\gamma_{i} \delta_{i}}, \quad\left|\delta_{i}\right| \leq 1 \\
k_{\text {pert }} & =\mathcal{F}_{L}\left(\left[\begin{array}{cc}
k_{\text {nominal }} & \gamma_{k} \\
1 & 0
\end{array}\right], \delta_{k}\right) \\
& =: \mathcal{F}_{L}\left(\mathcal{K}, \delta_{k}\right)=k_{\text {nominal }}+\gamma_{k} \delta_{k}, \quad\left|\delta_{k}\right| \leq 1
\end{aligned}
$$




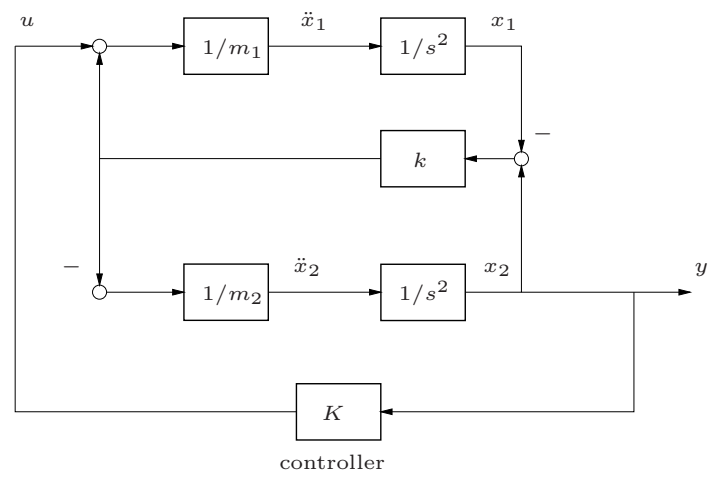

Figure 3: Detailed view onto the uncertain parameters within the benchmark system.

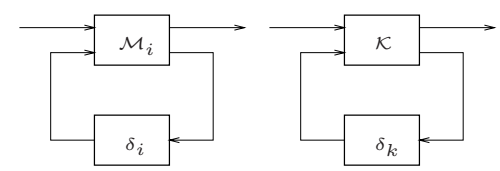

Figure 4: Replacing the uncertain parameters by appropriate LFTs.

Now, the uncertain parameters are represented by weighted additive errors to the nominal values

$$
\begin{aligned}
m_{i, \text { pert }} & =m_{i, \text { nominal }}+\gamma_{i} \delta_{i}, \quad\left|\delta_{i}\right| \leq 1, i=1,2 \\
k_{\text {pert }} & =k_{\text {nominal }}+\gamma_{k} \delta_{k}, \quad\left|\delta_{k}\right| \leq 1 .
\end{aligned}
$$

and the problem is formulated for $\mu$-analysis.

\section{Controller Design}

\subsection{Parameters for Design and Analysis}

One test for robustness demands stability for all $0.5 \leq k \leq 2.0$, so we will change the nominal value of the spring constant to the middle of this interval, 1.25 . Thus, the nominal values are:

$$
m_{1, \text { nominal }}=m_{2, \text { nominal }}=1, \quad k_{\text {nominal }}=1.25
$$

What about the admissible reference signals? The amplitude is given by the prescribed step-input. As admissible speed, we calculate the highest possible speed up to reaching the amplitude's bound 1 while stimulating the system with a step, which leads to :

$$
R=1, \quad \dot{R}=1.46
$$

The desired bound for the control variable is also mentioned in benchmark problem as

$$
u_{\max }^{\mathrm{des}}=1
$$

Design of a suboptimal controller (using a performance factor of $f=1.1$ is performed using the extended $\mathcal{H}_{\infty}$ loop-shaping design procedure.

\subsection{Choice of the weights}

We compare two different controllers, based on the constant weight $w_{1}=0.27$ resp. the non-constant weight $w_{2}(s)=\frac{0.31 s^{3}+1.42 s^{2}+7.21 s+5.66}{s^{3}+72.11 s^{2}+44.80 s+19.70}$. 


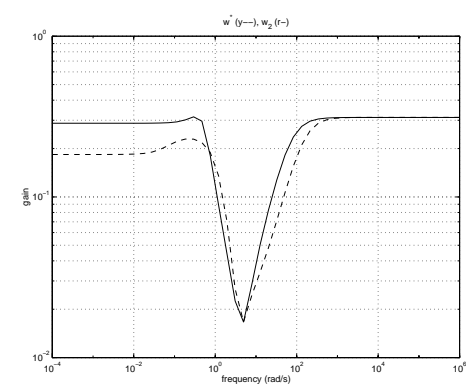

Figure 5: Design weights $w^{*}$ and $w_{2}$.

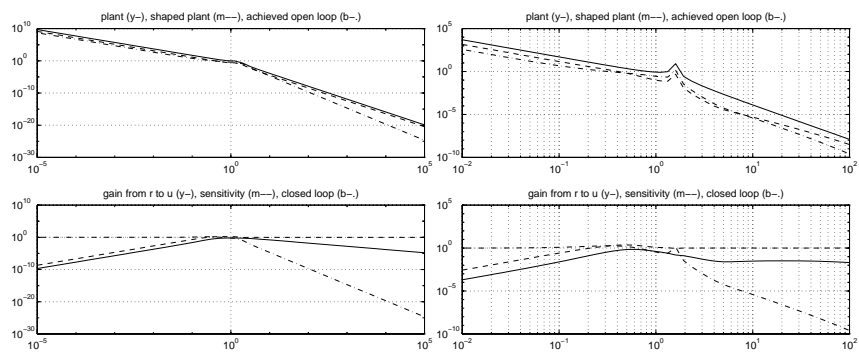

Figure 6: Design \#1 (left) and Design \#2 (right).

The choice of the constant weight $w_{1}$ is a simple and straightforward binary-search: choose $w_{1}$, so that the desired bound for the control signal is met. This binary search is possible because we are able to calculate the maximum control signal exactly .

The idea for the non-constant weight is using $w_{1}=0.27$ as a basic value and decrease it in the frequency range $10^{0} \ldots 10^{1}$, where the undamped eigenvalues of the plant appear, in order to damp them. Here, eqn.(2) is used to give a guideline for the weight adjustment: suppose, an initial weight $w^{*}$ is chosen to damp the system poles. The magnitude is shown in figure 5. It produces a control system with a maximum control signal of $u_{\max }^{*}=0.78$. But we can effort more! Consequently following the advice given in section 3 , we increase the weight in the frequency region $10^{-1} \ldots 10^{0}$, as the $\infty$-norm of the transfer function $H$ from $r$ to $u$ appears in this range. This leads, after some iterations, to the weight $w_{2}$, which is also given in figure 5 . It leads to a control system with $u_{\max }^{*}=0.96$. Fig. 6 shows the magnitudes of the important transfer functions.

\section{$5.3 \mu$-analysis}

In table 1 , results of a $\mu$-analysis are shown. For the presented tests, the parameter $\gamma=\left(\gamma_{1}, \gamma_{2}, \gamma_{k}\right)$ (cf. eqns $(3,4)$ ) for the relative change is varied until the upper bound for $\mu$, called $\mu_{h i}$, fulfills $\mu_{h i} \approx 1$ and $\mu_{h i}<1$. Obviously, Design \# 2 allows a larger variation of the parameters. We pay for this feature with a higher controller order, as a result from the higher weight order. 

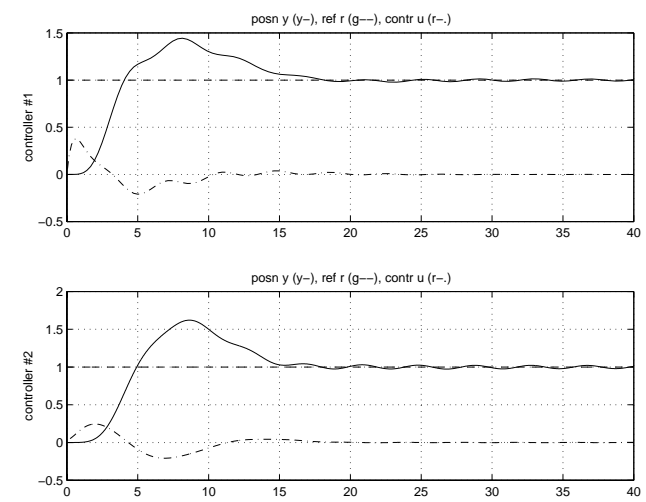

Figure 7: Simulation for nominal parameter set $m_{1}=m_{2}=1, k=1.25$.

\begin{tabular}{c|cc|cc} 
weight & $u_{\max }$ & $\epsilon$ & \multicolumn{3}{|c}{$\mu$-test: maximal parameters } \\
& & & \multicolumn{2}{|c}{$\gamma_{1}, \gamma_{2}, \gamma_{k}$ with $\mu \nearrow 1$} \\
\hline \hline$w_{1}=$ & 1.00 & 0.36 & vary all & $m_{i}$ fixed \\
0.27 & & & $0.1,0.1,0.27$ & $0,0,0.45$ \\
& & & $0.2,0.2,0.32$ & \\
& & & $0.5,0.5,0.03$ & \\
\hline & & & $k$ fixed & $m_{2}, k$ fixed \\
& & & $0.60,0.60,0$ & $0.90,0,0$ \\
\hline \hline$w_{2}$ & 0.96 & 0.27 & vary all & $m_{i}$ fixed \\
& & & $0.1,0.1,0.77$ & $0,0,1.20$ \\
& & & $0.2,0.2,0.82$ & \\
& & & $0.5,0.5,0.60$ & \\
\hline & & & $k$ fixed & $m_{2}, k$ fixed \\
& & & $0.65,0.65,0$ & $0.80,0,0$ \\
\hline
\end{tabular}

Table 1: Overview of the two designs.

\section{Selected Simulation Studies}

We simulate the step response (the recommended test for this benchmark problem) for selected parameter variations. We examine all relevant signals of the control loop. Notable, that in all simulation studies the bound for the control variable holds. As we are not stimulating the system with the worst case input, we are beyond the desired bound. We examine stability of the control loop when changing the parameters in their allowed ranges (see table 1) or even leaving these ranges slightly:

Experiment 1 - nominal plant: we simulate the two control systems using the nominal plant, see fig. 7 . We observe, that system \#2 has slightly larger overshoot, due to a lower control signal. On the other hand, it is more robust with respect to parameter changes as shown in table 1.

Experiment 2 - variation of $\mathbf{m}_{\mathbf{1}}$ : In the second study, shown in fig. 8, we only change the first mass $m_{1}$. A change of $80 \%$ is possible for all designs, which is clear from the table and shown in fig. 8 (left). But for design \#2, 80 percent are already the limit. We test this raising $m_{1}$ to 1.85 (i.e. a change of $85 \%$, and indeed, system \#2 is already unstable. The other way round, this means that the upper bound for $\mu$, as calculated in the $\mu$-test, is quite exact.

\section{Conclusions and Future Works}

We studied the control of control systems with hard bounded control signals, especially the two-mass-spring benchmark problem. One main point within the extension of the $\mathcal{H}_{\infty}$ Loops Shaping was the calculation of the maximum control signal for the set of admissible reference signals. The other main point was the 

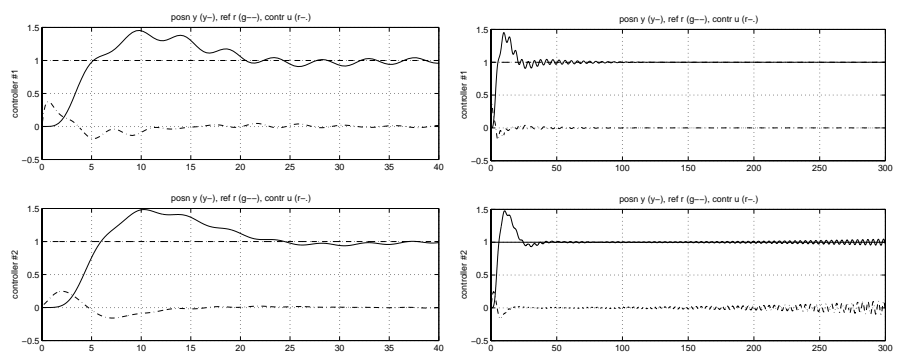

Figure 8: Simulation for parameter sets $m_{1}=1.8, m_{2}=1, k=1.25$ (left) and $m_{1}=1.85, m_{2}=1, k=1.25$ (right).

systematic adaption on the weights with respect to the control signals bound by deriving an explicit relation between design weight and maximum control signal..

Still an open question is how to guarantee the control signal within the suggested bounds for the set of uncertain plant - until now, this is only guaranteed for the nominal plant. We answered this question by an a-posteriori analysis. However, the simulation studies in this work showed sufficient behavior of the control signal even in the case of a parameter variation.

The extension of this work to the multivariable case is due to a future paper. Other future research will study hard constraints for other signals than the control signal, speed constraints for the control signal and applications with mixed hard and soft bounds.

\section{Acknowledgment}

Valuable discussions with Irina Zacharias during this work are gratefully acknowledged.

\section{References}

[1] D. S. Bernstein and A. N. Michel. A chronological bibliography on saturating actuators. Int. J. of Robust and Nonlinear Control, 5:375-380, 1995. Special Issue Saturating Actuators.

[2] R. D. Braatz and M. Morari. Robust control for a noncolocated spring-mass system. In Proc. of the American Control Conference, pages 2061-2062, Chicago, IL, USA, 1992.

[3] P. Dorato, R. Tempo, and G. Muscato. Bibliography on robust control. Automatica, 29(1):201-213, Jan. 1992.

[4] M. Green and D. J. N. Limebeer. Linear Robust Control. Prentice Hall, Englewood Cliffs, NJ, USA, 1995.

[5] M. V. Kothare. Control of Systems Subject to Constraints. PhD thesis, Dept of EE, California Institute of Technology, Pasadena, CA, USA, Mar. 1997.

[6] D. C. McFarlane and K. Glover. Robust Controller Design Using Normalized Coprime Factor Plant Description. Number 138 in Lecture Notes in Control and Information Science. Springer Verlag, Berlin, Germany, 1989.

[7] R. W. Reichel. Synthese von Regelsystemen mit Beschränkungen bei stochastischen Eingangsgrössen. PhD thesis, Dept of EE, University of Paderborn, 33095 Paderborn, Germany, 1984.

[8] W. Reinelt. $\mathcal{H}_{\infty}$ Loop Shaping for Systems with Hard Bounds. In Proc. of the Int Symp on Quantitative Feedback Theory and Robust Frequency Domain Methods, pages 89-103, Durban, South Africa, Aug. 1999. 
[9] W. Reinelt. Maximum output amplitude of linear systems for certain input constraints. Technical Report LiTH-ISY-R-2165, Dept of EE, Linköping University, Linköping, Sweden, June 1999. Accepted for presentation at CDC 2000 in revised and updated form, contained in technical report TR-2292.

[10] A. Saberi, A. A. Stoorvogel, and P. Sannuti. Control of Linear Systems with Regulation and Input Constraints. Communications and Control Engineering. Springer Verlag, London, UK, 2000.

[11] G. Vinnicombe. Robust design in the graph topology; a benchmark example. In Proc. of the American Control Conference, pages 2063-2064, Chicago, IL, USA, 1992.

[12] B. Wie and D. S. Bernstein. Benchmark problems for robust control design. Journal of Guidance, Control and Dynamics, 1(5):1057-1059, Sep/Oct 1992.

[13] K. Zhou, J. C. Doyle, and K. Glover. Robust and Optimal Control. Prentice Hall, Upper Saddle River, NJ, USA, 1996. 